\title{
Salmon cartilage proteoglycan attenuates allergic responses in mouse model of papain-induced respiratory inflammation
}

\author{
HISAYA K. ONO ${ }^{1}$, SAYURI YOSHIMURA ${ }^{2}$, SHOUHEI HIROSE ${ }^{3,4}$, KOUJI NARITA $^{3,5}$, \\ MAKOTO TSUBOI ${ }^{6}$, KRISANA ASANO ${ }^{3,4}$ and AKIO NAKANE ${ }^{3,4}$
}

\begin{abstract}
${ }^{1}$ Laboratory of Zoonoses, Kitasato University School of Veterinary Medicine, Towada, Aomori 034-8628; ${ }^{2}$ Tohoku Women's College, Hirosaki, Aomori 036-8503; ${ }^{3}$ Department of Microbiology and Immunology; ${ }^{4}$ Department of Biopolymer and Health Science;

${ }^{5}$ Institute for Animal Experimentation, Hirosaki University Graduate School of Medicine, Hirosaki, Aomori 036-8562;

${ }^{6}$ Research and Development Department, Ichimaru Pharcos Co., Ltd., Motosu, Gifu 501-0475, Japan
\end{abstract}

Received January 4, 2018; Accepted July 13, 2018

DOI: $10.3892 / \mathrm{mmr} .2018 .9364$

\begin{abstract}
Proteoglycan (PG) is a complex glycohydrate, which is widely distributed in the extracellular matrix. It has been reported that daily oral administration of PG (extracted from salmon nasal cartilage) modulates the severity of proinflammatory cytokine responses in mouse experimental colitis, autoimmune encephalomyelitis, collagen-induced arthritis and obesity-induced inflammation. The present study investigated the effect of salmon nasal cartilage PG on allergic responses using a mouse model of papain-induced respiratory inflammation. Low titers of immunoglobulin $\mathrm{E}$ were identified in the sera of the PG-administered mice. Oral administration of PG attenuated eosinophil infiltration in the lung. In the acute model of papain-induced allergic inflammation, PG-administered mice exhibited low titers of epithelium-derived and $\mathrm{T}$ helper 2 -associated cytokines. The results of the present study demonstrated that salmon cartilage PG has an immunomodulatory effect on intranasally delivered papain. These results suggest a potential role for PG as a prophylactic agent which may attenuate allergic respiratory inflammation.
\end{abstract}

\section{Introduction}

Proteoglycan (PG) is a natural biopolymer consisting of a core protein covalently attached to one or more glycosaminoglycan

Correspondence to: Dr Krisana Asano or Dr Akio Nakane, Department of Microbiology and Immunology, Hirosaki University Graduate School of Medicine, 5 Zaifu-cho, Hirosaki, Aomori 036-8562, Japan

E-mail: krisana@hirosaki-u.ac.jp

E-mail: a27k03n0@hirosaki-u.ac.jp

Abbreviations: BALF, bronchoalveolar lavage fluid; EAE, autoimmune encephalomyelitis; ELISA, enzyme-linked immunosorbent assay; IgE, immunoglobulin E; IL, interleukin; ILC2s, type 2 innate lymphoid cells; PG, proteoglycan; TSLP, thymic stromal lymphopoietin

Key words: proteoglycan, salmon cartilage, prophylactic agent, allergy, asthma chains. PG forms a complex with collagen, fibronectin, laminin, hyaluronic acid and other glycoproteins in the extracellular matrix and exists in the connective tissues, such as the skin, bone, cartilage and vascular wall of humans and animals (1). By incorporation with collagen, fibronectin and laminin, PG has been shown to be involved in cellular proliferation and adhesion (2). Regarding its complex structure and biological activity, PG has a high potential for medical and industrial applications. Nasal cartilage, a by-product accumulated from salmon (Oncorhynchus keta) consumption, is an attractive source of PG. PG from salmon nasal cartilage has been purified and characterized $(3,4)$. It has been identified as an aggrecan with molecular weight of approximately $1,400,000$ daltons (4). Its core protein comprises 1,324 amino acids with a molecular weight of 143,276 daltons. Based on its amino acid sequence, 46 serine residues are estimated to attach to sugar chains and the disaccharide composition in these sugar chains contains $60 \%$ of chondroitin- 6 sulfate (3). Our previous studies have demonstrated that salmon PG has an immunomodulatory effect (5-9). Salmon PG suppresses inflammatory responses from mouse macrophages induced by heat-killed Escherichia coli (5). In mouse experimental models, daily oral administration of salmon PG attenuates the severity of inflammatory colitis (6), autoimmune encephalomyelitis (EAE) (7), collagen-induced arthritis (8) and obesity-induced inflammation (9). The attenuation of systemic inflammation in colitis and EAE models by daily oral administration of PG is associated with a reduction in T helper (Th)17 lineage differentiation and enhancement of Foxp3 $3^{+}$regulatory T cells $(6,7)$.

The systemic immunomodulatory effect of $\mathrm{PG}$ has prompted us to investigate the effect of PG on immune responses in mouse models of allergic asthma. In the present study, the cysteine protease papain was used as a sensitizing agent for inducing allergic airway inflammation. In allergic asthma, dendritic cells present allergens to $\mathrm{CD} 4^{+} \mathrm{T}$ cells, inducing $\mathrm{Th} 2$ cells to produce interleukin (IL)-4, IL-5 and IL-13, which then lead to immunoglobulin E (IgE) switching in B cells, airway eosinophilia and mucous hypersecretion (10). In addition, certain air pollutants, microbes and glycolipids are able to induce the release of epithelium-derived cytokines, including thymic stromal lymphopoietin (TSLP). This cytokine activates 
type 2 innate lymphoid cells (ILC2s) to produce high amounts of IL-5 and IL-13, leading to eosinophilia, mucous hypersecretion and airway hyperreactivity (11).

In the present study, serum IgE, Th2-related cytokines, TSLP and eosinophils in bronchoalveolar lavage fluid (BALF) were evaluated following papain administration. The effect of salmon PG was investigated, as was whether daily oral administration of PG attenuated these allergic airway inflammatory responses.

\section{Materials and methods}

Preparation of mice. C57BL/6 mice were purchased from Clea Japan, Tokyo, Japan. They were maintained under specific pathogen-free conditions with a temperature-controlled room $\left(22^{\circ} \mathrm{C}\right)$ on a 12 -h light-dark cycle at the Institute for Animal Experimentation, Hirosaki University Graduate School of Medicine. All animal experiments were carried out in accordance with the Institutional Animal Care and Use Committees/ethics Committee of Hirosaki University. The study was approved by the Ethics Committee of the Institute for Animal Experimentation, Hirosaki University Graduate School of Medicine (approval no. M08028).

Sensitization and challenge of mice with papain. Mice were sensitized and challenged with papain as described previously $(12,13)$, with minor modifications. Briefly, papain (Sigma-Aldrich; Merck KGaA, Darmstadt, Germany) was dissolved in phosphate-buffered saline (PBS). Mice (6 to 12-week old) were sedated by intraperitoneal injection with a mixture of anesthetic agents $[0.075 \mathrm{mg} / \mathrm{ml}$ medetomidine (Zenoaq, Tokyo, Japan), $0.4 \mathrm{mg} / \mathrm{ml}$ midazolam (Sandoz, Tokyo, Japan) and $0.5 \mathrm{mg} / \mathrm{ml}$ butorphanol (Meiji Seika Pharma Co., Ltd., Tokyo, Japan)] at $100 \mu \mathrm{l} / 10 \mathrm{~g}$ body weight and allowed to inhale papain $(1 \mu \mathrm{g}$ in $40 \mu \mathrm{l})$ into both nostrils (20 $\mu \mathrm{l}$ each). Inhalation of PBS $(40 \mu \mathrm{l})$ was performed in the control group. The sedated mice were reversed by intraperitoneal injection with $0.075 \mathrm{mg} / \mathrm{ml}$ of atipamezol hydrochloride (Antisedan; Zenoaq) at $100 \mu \mathrm{l} / 10 \mathrm{~g}$ body weight. The intranasal administration of papain was performed daily for 5 consecutive days. After 2 weeks, mice were boosted using the same regimen. A total of 2 weeks post-boost, mice were challenged by daily intranasal delivery of papain $(100 \mu \mathrm{g}$ in $40 \mu \mathrm{l}$ ) for 3 consecutive days. At $24 \mathrm{~h}$ after the final challenge, mice were euthanized. Serum and BALF were collected for further analysis. To accelerate acute allergic responses, mice were administered intranasally as described above with $10 \mu \mathrm{g}$ papain (in $40 \mu \mathrm{l}$ ) on days 0 and 7 . At $6,12,24$ and $72 \mathrm{~h}$ after the second sensitization with papain, all mice were euthanized. Then, the serum and BALF were collected.

$P G$ preparation and administration. $\mathrm{PG}$ extracted from salmon nasal cartilage was purchased from Kakuhiro Co., Ltd., (Aomori, Japan). It was added into drinking distilled water (DW) at a concentration of $0.2 \mathrm{mg} / \mathrm{ml}$ and the mice drank ad libitum by starting on the first papain-sensitized day. DW only was used as a negative control. A mouse drank approximately $5 \mathrm{ml} /$ day. Thus, the consumption of $\mathrm{PG}$ was estimated at $1 \mathrm{mg} / \mathrm{day}$.

Analysis of serum $\operatorname{IgE}$. The titer of total IgE in the serum was determined by sandwich enzyme-linked immunosorbent assay (ELISA) using purified rat anti-mouse $\mathrm{IgE}$ monoclonal antibody (catalog number 1130-01; Southern Biotechnology Associates, Inc., Birmingham, AL, USA) as a capture reagent, whereas heat-inactivated papain was used to determine papain-specific IgE. For detection, goat anti-mouse IgE antibody conjugated with horseradish peroxidase (cat. no. 1110-05; Southern Biotechnology Associates, Inc.) was used. Mouse IgE isotype control (cat. no. 0114-01; Southern Biotechnology Associates, Inc.) was used to establish a standard curve for total IgE. On the other hand, a pool of sera with a high titer of papain-specific IgE was used as a standard for the specific IgE. The optical density obtained from 100 times-diluted standard serum was assigned the arbitrary unit of 1.0. The titer of papain-specific IgE in the samples was determined as a relative value to the standard IgE-positive serum.

Collection of BALF and analysis of BALF cells. After anesthetization of the mice, BALF was collected via an incision to the trachea $(12)$. PBS $(0.5 \mathrm{ml})$ was flushed into the lungs and recovered using $1 \mathrm{~mm}$ diameter polyethylene tubing and a $1 \mathrm{ml}$ syringe. BALF cells were collected by centrifugation at $100 \mathrm{x} \mathrm{g}$ at $4^{\circ} \mathrm{C}$ for $10 \mathrm{~min}$ and suspended in $40 \mu \mathrm{l}$ of PBS. BALF cells were spread on a glass slide and Giemsa staining was performed according to the standard protocol. The granulocytes were then counted under a light microscope (Olympus CX31; Olympus Corporation, Tokyo, Japan) and the proportion of eosinophils was evaluated from the number of granulocytes. The number of granulocytes per observed area was in the range of 100-148. BALF was collected and stored at $-20^{\circ} \mathrm{C}$ until use.

Histological analysis of lung tissue. A total of $72 \mathrm{~h}$ following the second sensitization of papain in the acute allergic model, mice were euthanized. Lung tissue was collected and fixed in $4 \%(\mathrm{w} / \mathrm{v})$ paraformaldehyde buffer at $4^{\circ} \mathrm{C}$ overnight. Tissues were then embedded in paraffin and cut into $5-\mu \mathrm{m}$ thick sections. Deparaffinized sections were stained with hematoxylin and eosin. The tissues were observed under a BZ-X700 microscope (Keyence, Tokyo, Japan). Infiltrated cells were randomly counted from four histological sections.

Detection of cytokines in BALF and serum. The titer of TSLP, IL-4, IL-13 and IL-5 in the BALF and serum was determined using ELISA kits, according to the manufacturer's protocol. Determination of TSLP and IL-13 production was achieved using a Mouse TSLP ELISA Ready-SET-Go! ${ }^{\mathrm{TM}}$ kit (cat. no. 5017406; Thermo Fisher Scientific, Inc., Waltham, MA, USA) and Novex ${ }^{\mathrm{TM}}$ IL13 Mouse Antibody Pair (cat. no. 10180373; Thermo Fisher Scientific, Inc.), respectively. The production of IL-4 and IL-5 was determined using Mouse IL-4 Antibody Pair and Mouse IL-5 CytoSet ${ }^{\mathrm{TM}}$, respectively (cat. nos. CMC0043 and CMC0053; Invitrogen; Thermo Fisher Scientific, Inc.).

Statistical analysis. Data are expressed as the mean \pm standard deviation. Statistical analyses were performed using Ystat 2018 software (Igaku Tosho Shuppan Co., Ltd., Tokyo, Japan) together with Microsoft Excel software (Microsoft Corporation, Redmond, WA, USA). The statistical significance between two samples was performed by a Mann-Whitney 

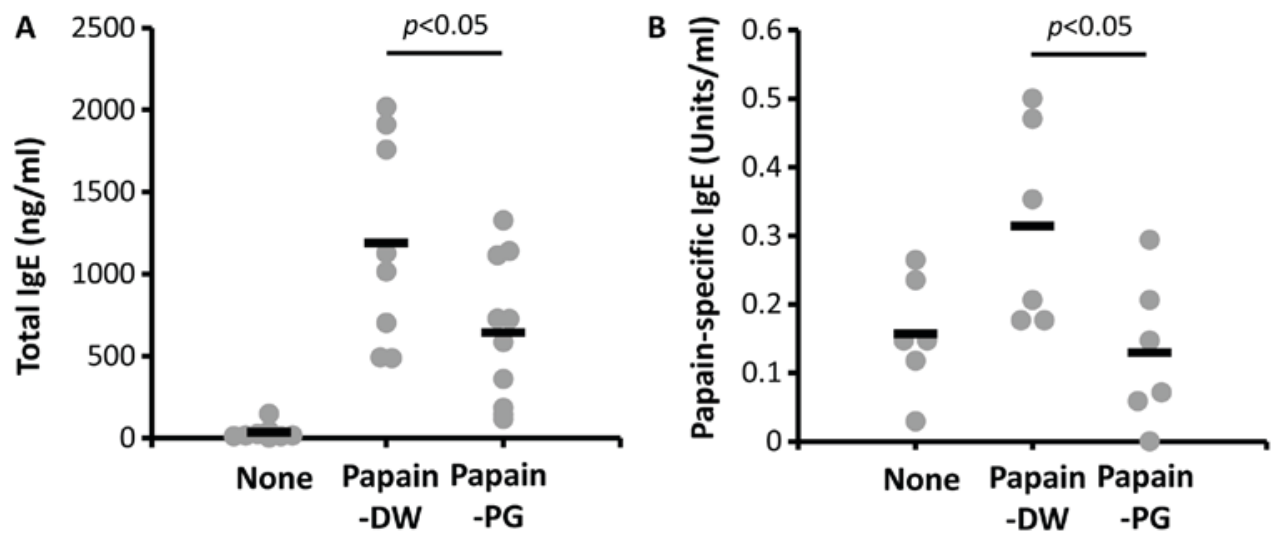

Figure 1. Effect of PG on an IgE in a mouse model of papain-induced allergy. (A) Mice were administered $10 \mu \mathrm{g}$ papain intranasally on days 0 and 7 . A total of $24 \mathrm{~h}$ later the serum was collected and total IgE was measured using ELISA. (B) Mice were sensitized with $1 \mu \mathrm{g}$ papain for 5 days. After 2 weeks, the mice were boosted using the same regimen. Two weeks post-boost, the mice were challenged by daily intranasal delivery of $100 \mu \mathrm{g}$ papain for 3 consecutive days A total of $24 \mathrm{~h}$ later the serum was collected and papain-specific IgE were determined by ELISA. Each dot on the graph represents the value of an individual mouse. The horizontal bar indicates the mean value for each group. IgE, immunoglobin E; None, naïve mice; PG, proteoglycan; DW, distilled water.

U-test, whereas ANOVA analysis with a post-hoc Tukey's test was used for multiple comparisons. $\mathrm{P}<0.05$ was considered to indicate a statistically significant difference.

\section{Results}

Salmon PG suppresses serum IgE levels in papain-sensitized mice. To investigate the effect of PG on allergic responses in papain-sensitized mice, serum $\operatorname{IgE}$ levels were determined. As presented in Fig. 1, the IgE level of papain-sensitized mice with DW-drinking increased after challenging with papain. The serum IgE of PG-administered mice was significantly decreased compared with mice with DW-drinking. This result is promising for the further examination of the suppressive effect of PG in allergy mouse models.

Salmon PG suppresses eosinophil infiltration in lung. To observe the effect of PG on eosinophil infiltration in the lung, the cells in the BALF were collected and counted. In the naive mice, the infiltration of eosinophils could not be detected. Conversely, there was increased infiltration of eosinophils into the BALF following papain sensitization and challenge for 3 consecutive days. This infiltration significantly decreased when mice were orally administered with PG (Fig. 2A and B). To accelerate the acute allergic response, mice were administered intranasally with $10 \mu \mathrm{g}$ papain on days 0 and 7. In this acute model, the effect of PG on eosinophil infiltration was also confirmed. PG reduced eosinophil infiltration in the BALF from $12 \mathrm{~h}$ after the second sensitization, and the effect was retained for up to 3 days (Fig. 2C).

$P G$ reduces perivascular cell infiltration. The histology of the lung tissue was then analyzed to investigate the effect of PG on lung tissue damage. Lung tissue damage was not observed in papain-sensitized mice. However, the papain-sensitized mice with DW-drinking had an increased number of inflammatory cells infiltrating around the alveolar septa, compared with the control mice. Conversely, lung inflammation was reduced by salmon PG. Almost no inflammatory cell infiltration was observed in the papain-sensitized mice with PG-administration (Fig. 3A). Fig. 3B shows the quantitative analysis of cell infiltration in the lung tissue.

$P G$ suppresses the production of an epithelium-derived cytokine. Epithelium-derived cytokines have been known to activate $\mathrm{T}$ cells in an antigen-dependent manner and ILC2s in an antigen-independent manner. Thus, production of the epithelium-derived cytokine TSLP was examined in acute allergic-induced mice. At $12 \mathrm{~h}$ after the second sensitization, the titer of TSLP in the BALF and serum was determined by ELISA. Although the titer of TSLP of papain-sensitized mice in the BALF was low and PG had no effect, PG suppressed TSLP production in the serum (Fig. 4).

$P G$ suppresses Th2-related cytokines in the BALF and serum. Th2 cells are known to drive asthma pathobiology, thus titers of Th2-related cytokines were evaluated. At $12 \mathrm{~h}$ after the second sensitization of acute allergic-induced mice, BALF and serum were collected and the titers of IL-4, IL-5 and IL-13 were determined by ELISA. The results showed that both IL-4 and IL-13 could not be detected in the serum (Fig. 5A and B). Although IL-4 production was detected at low levels in the BALF, PG had no significant effect (Fig. 5A). In contrast, the production of IL-13 in the BALF could be detected in papain-sensitized mice and PG suppressed this IL-13 production (Fig. 5B). The high titer of IL-5 was detected in both the serum and BALF of papain-sensitized mice. The production of IL-5 in PG-administered mice was significantly lower compared with DW-drinking control mice (Fig. 5C).

\section{Discussion}

At present, foods are intended not only to provide necessary nutrients, however also to prevent nutrition-related diseases and improve physical and mental wellbeing $(14,15)$. In this regard, the development of functional foods and dietary supplements is a focus, in order to improve healthcare and disease prevention (16-19). Salmon nasal cartilage is a major by-product of salmon consumption (20). PG extracted from salmon nasal 
A

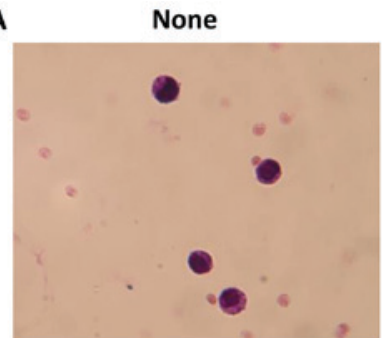

B

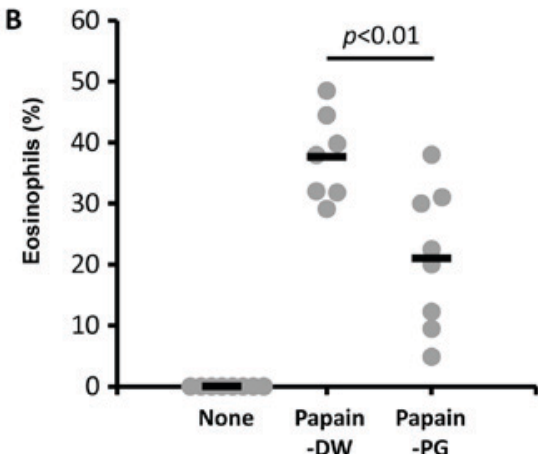

Papain-DW
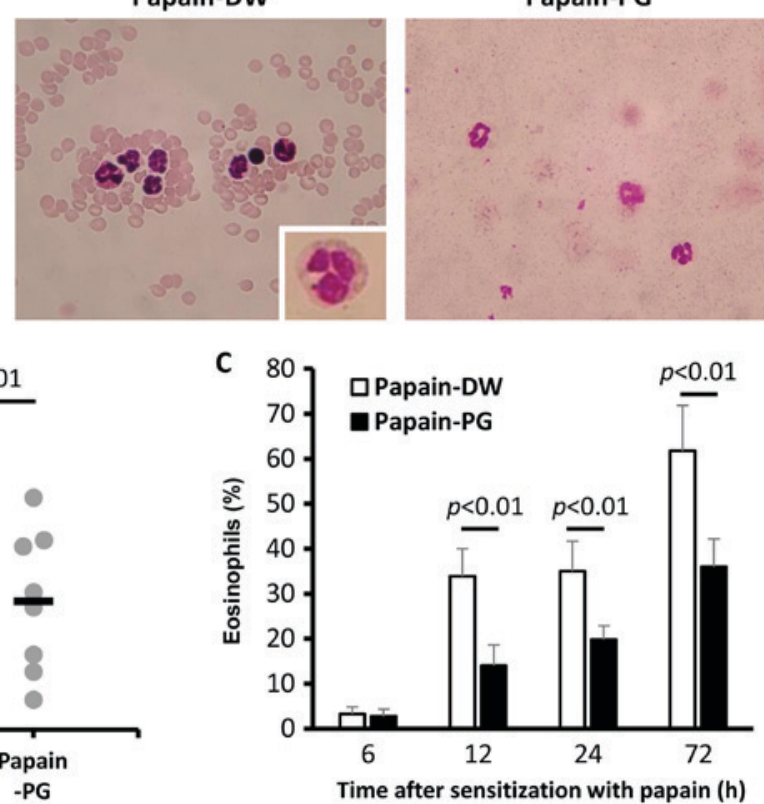

Figure 2. Effect of PG on eosinophil infiltration in papain-sensitized mice. Mice were sensitized daily with $1 \mu \mathrm{g}$ papain for 5 days. After two weeks the mice were boosted using the same regimen. A total of 2 weeks post-boost the mice were challenged by daily intranasal delivery of $100 \mu \mathrm{g}$ papain for 3 consecutive days. A total of $24 \mathrm{~h}$ later, the BALF was collected and (A) BALF cells were stained with Giemsa. Magnification, $x 40$. Images are representative of 3-6 mice from each group. Inset, enlargement of an eosinophil. (B) BALF cells were counted and the percentage of eosinophils was calculated from the number of granulocytes. Each dot on the graph represents the value of an individual mouse. The horizontal bar indicates the mean value for each group. (C) Mice were administered $10 \mu \mathrm{g}$ papain intranasally on days 0 and 7 . At 6, 12, 24 and $72 \mathrm{~h}$ after the second sensitization with papain, the BALF was collected. BALF cells were stained with Giemsa and counted. The percentage of eosinophils was calculated from the number of granulocytes. BALF, bronchoalveolar lavage fluid; None, naïve mice; PG, proteoglycan; DW, distilled water.
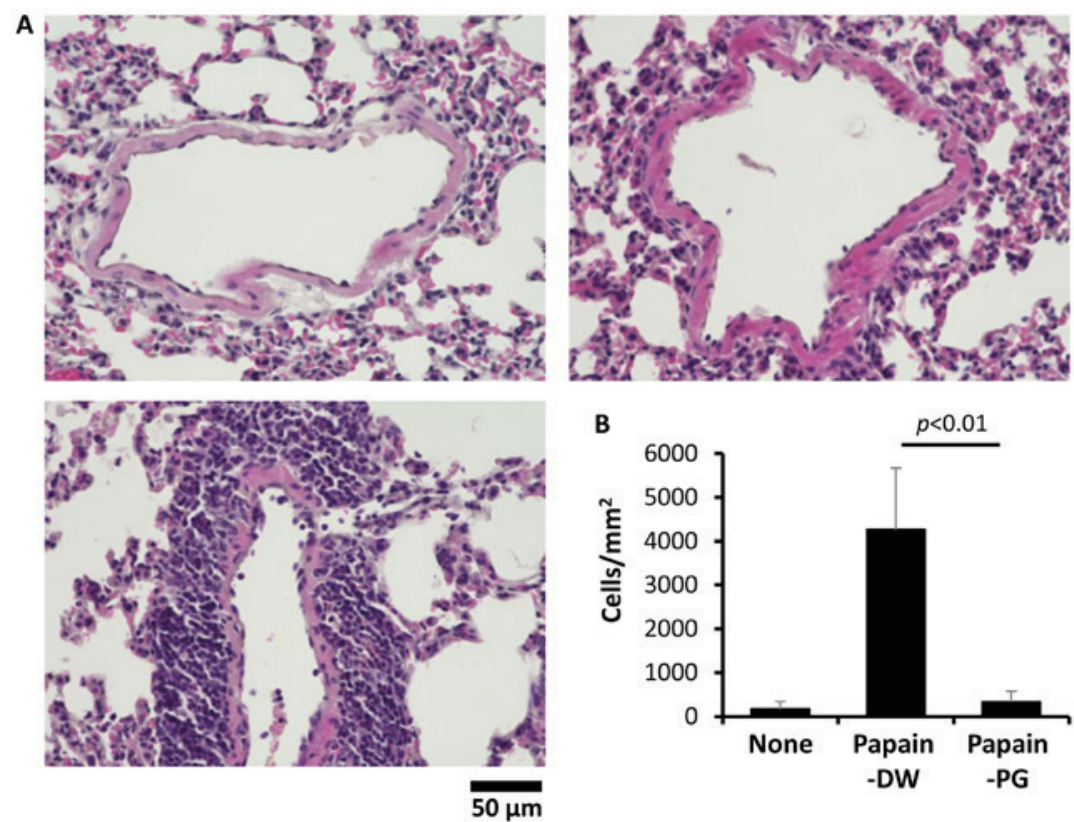

Figure 3. Effect of PG on the lung tissue of papain-sensitized mice. Mice were administered $10 \mu \mathrm{g}$ papain intranasally on days 0 and 7 . A total of $72 \mathrm{~h}$ after the second sensitization with papain, lung tissue was collected and histological analysis was performed. (A) Images are representative of 3 mice of each group. Upper left, tissue from naïve mice; lower left, tissue from papain-sensitized mice with DW control; upper right, tissue from papain-sensitized mice with PG administration (hemaoxylin and eosin staining). (B) Quantitative analysis of cell infiltration in the lung tissue is shown. PG, proteoglycan; DW, distilled water.

cartilage is a complex biopolymer which has the potential to be a high-value biomaterial. Several properties of salmon PG have been characterized. Salmon PG has been shown to exhibit immunomodulatory effects in both in vitro and in vivo experiments (5-9). However, daily oral administration of salmon PG
( $2 \mathrm{mg} /$ day) for up to 3 months did not promote anti-PG antibody production or infection susceptibility (data not shown). This property makes PG to become an attractive prophylactic agent for use as a supplement. In the present study, the effect of PG on allergic responses in a mouse model of papain-induced 

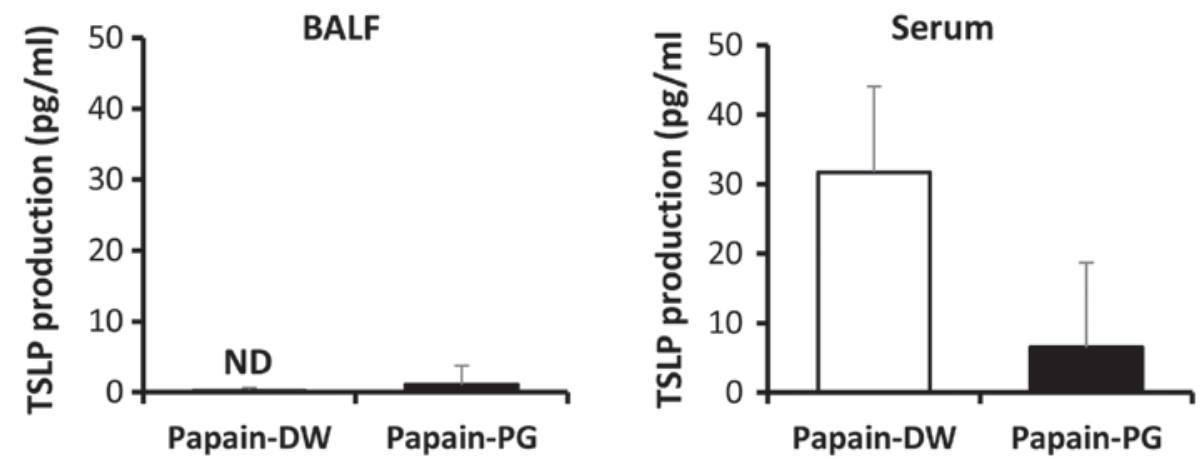

Figure 4. Effect of PG on the production of an epithelium-derived cytokines. Mice were administered $10 \mu \mathrm{g}$ papain intranasally on days 0 and 7 . A total of $12 \mathrm{~h}$ after the second sensitization with papain the BALF and serum were collected. The titers of TSLP $(n=6)$ were determined by ELISA. ND, not detected; BALF, bronchoalveolar lavage fluid; PG, proteoglycan; DW, distilled water; TSLP, thymic stromal lymphopoietin.

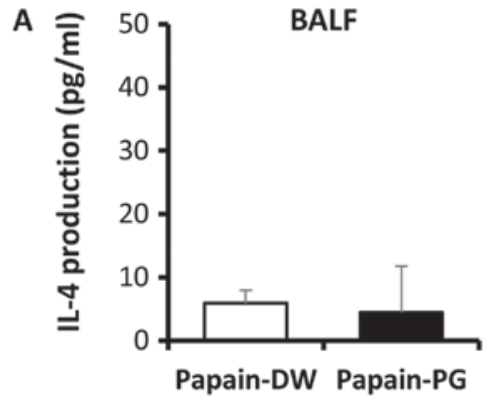

B

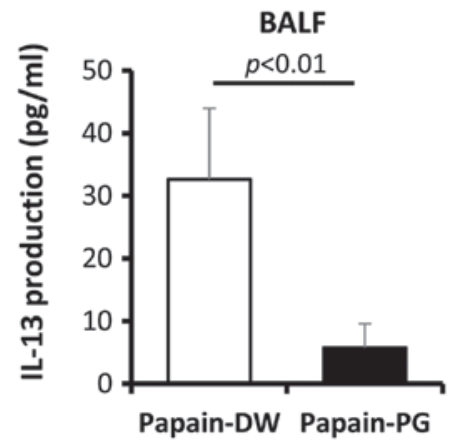

C

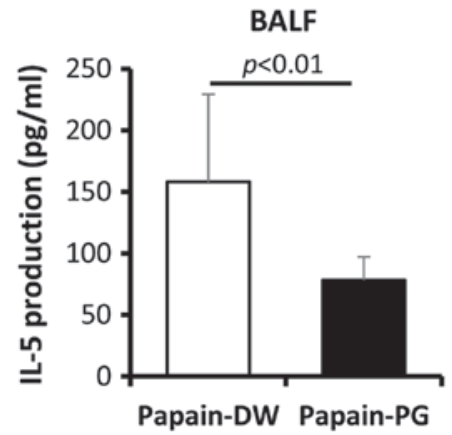

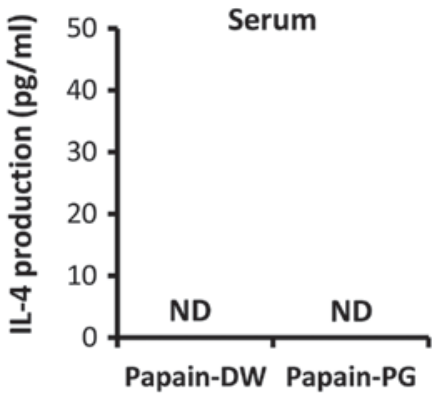

Serum
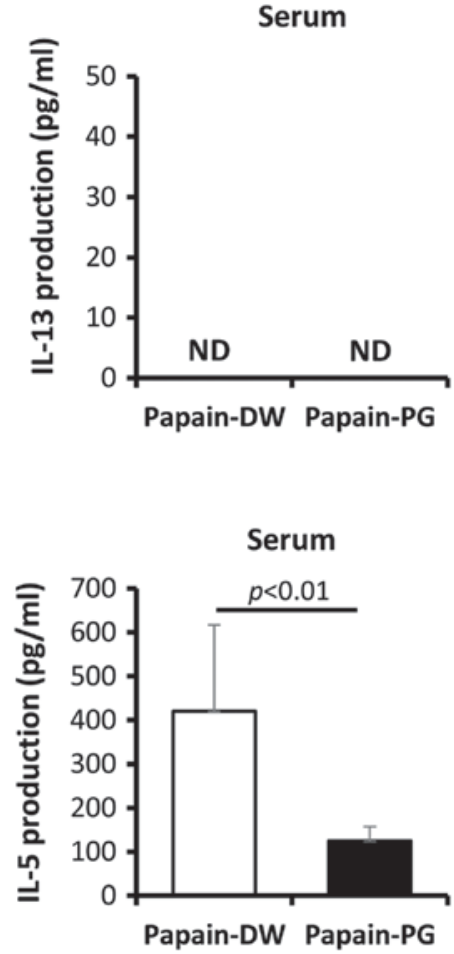

Figure 5. Effect of PG on the production of T helper 2-associated cytokines. Mice were administered $10 \mu \mathrm{g}$ papain intranasally on days 0 and 7. A total of $12 \mathrm{~h}$ after the second sensitization with papain, the BALF and serum were collected. The titers of (A) IL-4 ( $\mathrm{n}=3$ ), (B) IL-13 ( $\mathrm{n}=7)$ and (C) IL-5 ( $\mathrm{n}=10)$ were determined by ELISA. ND, not detected; BALF, bronchoalveolar lavage fluid; IL, interleukin; PG, proteoglycan; DW, distilled water.

respiratory inflammation was investigated. The amount of PG and the starting time of administration were designed based on our previous studies (6-9).

Allergies are defined as an overreaction of the immune system to allergens such as pollen or certain foods (21). This disease is widespread and affects a large number of people worldwide. Allergens stimulate plasma cells to produce IgE and bind IgE that has attached to mast cells. This binding triggers the release of histamine from mast cells. In the lung, histamine causes bronchiole constriction and airflow reduction, 
called asthma (22). Although allergies in specific tissues are not life-threatening, the allergic response in the bloodstream can be fatal. To attenuate allergies, the use of prophylactic agents is an alternative method to allergen avoidance. To demonstrate whether salmon PG has an immunomodulatory effect on allergies, a mouse model of papain-induced allergic asthma was used in the present study. Papain, a cysteine protease, is a homolog of the major house dust mite allergen Dermatophagoides pteronyssinus Der p $1(12,13)$. It can induce respiratory inflammation by intranasal administration with $1 \mu \mathrm{g}$ several times (12) or with $10 \mu \mathrm{g}$ twice (13). From both models of papain administration, we demonstrated that eosinophil infiltration in the lung significantly increased in comparison to naïve mice (Fig. 2).

Th2 cytokines play important roles in allergic asthma. These cytokines, such as IL-4, IL-5 and IL-13, are thought to be related to Th2 immunity (10). Conversely, epithelium derived-cytokines such as IL-33 and TSLP play an important role in non-allergic airway inflammation (11). These cytokines activate ILC2s to produce IL-5 and IL-13, however not IL-4. Of the Th2 cytokines, IL- 4 and IL-13 drive naïve Th cells toward the Th2 phenotype and induce B cells to switch their isotype to IgE, whereas IL-5 produced by Th2 cells is responsible for eosinophil growth, differentiation, mobilization, recruitment, activation and survival. Excessive production of IL-4, IL-5 and IL-13 has been implicated in the development of asthma (22). In the present study, intranasal papain administration increased TSLP production in the serum (Fig. 4) and T-cell responsive Th2 cytokines, IL-5 and IL-13 in the BALF (Fig. 5). For an unknown reason, the production of IL-4 and TSLP was low. This may have been due to the lower susceptibility of C57BL/6 mice to papain sensitization, in comparison with that of BALB/c mice. However, PG significantly suppressed the production of TSLP in the serum, and IL-5 and IL-13 in the BALF (Figs. 4 and 5) which are expected to suppress eosinophil infiltration and IgE-switching in B cells. In this regard, salmon PG attenuated perivascular cell infiltration (Fig. 3). To further confirm the effect of PG on allergic response, the airway hyperresponsiveness which is a common finding in allergic bronchial inflammation is required.

The molecular mechanism of PG and its immunomodulatory effect remains to be determined. The effect of PG on intestinal microbiota is one of several possibilities. Daily oral administration of salmon PG has been shown to improve intestinal microbiota by enhancing probiotics and short chain fatty acid-producing bacteria (23). Alternatively, salmon PG may directly affect the intestinal epithelium or immune cells, which then regulate intestinal and systemic immune responses.

In conclusion, the present study demonstrated that salmon cartilage PG attenuates allergic responses in a mouse model of papain-induced respiratory inflammation. Oral administration of PG reduced the serum IgE, eosinophil infiltration and the titers of epithelium-derived and Th2-related cytokines. Although only a partial ability of PG to relieve acute allergic asthma was revealed, and not a chronic reaction, the data in the present study suggested that continual consumption of salmon PG may be able to attenuate overreactive allergies.

\section{Acknowledgements}

Not applicable.

\section{Funding}

The present study was supported by the City Area Program for the Promotion of Science and Technology in Regional Areas from the Ministry of Education, Culture, Sports, Science and Technology.

\section{Availability of data and materials}

The datasets used and/or analyzed during the current study are available from the corresponding author upon reasonable request.

\section{Authors' contributions}

AN conceived and designed the study. AN, KA and MT conceived the study and were involved in funding acquisition. HKO, SY, SH and KN performed the experiments and analyzed the data. KA wrote and revised the manuscript. All authors read and approved the final manuscript.

\section{Ethics approval and consent to participate}

The study was approved by the Ethics Committee of the Institute for Animal Experimentation, Hirosaki University Graduate School of Medicine (approval no. M08028).

\section{Patient consent for publication}

Not applicable.

\section{Competing interests}

The authors declare that they have no competing interests.

\section{References}

1. Har-el R and Tanzer ML: Extracellular matrix. 3: Evolution of the extracellular matrix in invertebrates. FASEB J 7: 1115-1123, 1993.

2. Danen EH and Yamada KM: Fibronectin, integrins, and growth control. J Cell Physiol 189: 1-13, 2001.

3. Kakizaki I, Mineta T, Sasaki M, Tatara Y, Makino E and Kato Y: Biochemical and atomic force microscopic characterization of salmon nasal cartilage proteoglycan. Carbohydr Polym 103: 538-549, 2014.

4. Kakizaki I, Tatara Y, Majima M, Kato Y and Endo M: Identification of proteoglycan from salmon nasal cartilage. Arch Biochem Biophys 506: 58-65. 2011.

5. Sashinami H, Takagaki K and Nakane A: Salmon cartilage proteoglycan modulates cytokine responses to Escherichia coli in mouse macrophages. Biochem Biophys Res Commun 351: 1005-1010, 2006.

6. Mitsui T, Sashinami H, Sato F, Kijima H, Ishiguro Y, Fukuda S, Yoshihara S, Hakamada K and Nakane A: Salmon cartilage proteoglycan suppresses mouse experimental colitis through induction of Foxp $3^{+}$regulatory T cells. Biochem Biophys Res Commun 402: 209-215, 2010.

7. Sashinami H, Asano K, Yoshimura S, Mori F, Wakabayashi K and Nakane A: Salmon proteoglycan suppresses progression of mouse experimental autoimmune encephalomyelitis via regulation of Th17 and Foxp3(+) regulatory T cells. Life Sci 91: 1263-1269, 2012.

8. Yoshimura S, Asano $\mathrm{K}$ and Nakane A: Attenuation of collagen-induced arthritis in mice by salmon proteoglycan. Biomed Res Int 2014: 406453, 2014.

9. Hirose S, Asano K and Nakane A: Attenuation of obesity-induced inflammation in mice orally administered with salmon cartilage proteoglycan, a prophylactic agent. Biochem Biophys Res Commun 484: 480-485, 2017. 
10. Wenzel SE: Asthma phenotypes: The evolution from clinical to molecular approaches. Nat Med 18: 716-725, 2012.

11. Brusselle GG, Maes T and Bracke KR: Eosinophils in the spotlight: Eosinophilic airway inflammation in nonallergic asthma. Nat Med 19: 977-979, 2013

12. McGlade JP, Gorman S, Lenzo JC, Tan JW, Watanabe T, Finlay-Jones JJ, Thomas WR and Hart PH: Effect of both ultraviolet $\mathrm{B}$ irradiation and histamine receptor function on allergic responses to an inhaled antigen. J Immunol 178: 2794-2802, 2007.

13. Halim TY, Steer CA, Mathä L, Gold MJ, Martinez-Gonzalez I, McNagny KM, McKenzie AN and Takei F: Group 2 innate lymphoid cells are critical for the initiation of adaptive Thelper 2 cell-mediated allergic lung inflammation. Immunity 40: 425-435, 2014.

14. Roberfroid MB: Global view on functional foods: European perspectives. Br J Nutr 88 (Suppl 2): S133-S138, 2002.

15. Menrad K: Market and marketing of functional food in Europe. J Food Eng 56: 181-188, 2003.

16. Tang ML: Probiotics and prebiotics: Immunological and clinical effects in allergic disease. Nestle Nutr Workshop Ser Pediatr Program 64: 219-238, 251-257, 2009.
17. Floch $\mathrm{MH}$ and Hong-Curtiss J: Probiotics and functional foods in gastrointestinal disorders. Curr Gastroenterol Rep 3: 343-350, 2001.

18. Cencic A and Chingwaru W: The role of functional foods, nutraceuticals, and food supplements in intestinal health. Nutrients 2 : 611-625, 2010.

19. Alissa EM and Ferns GA: Functional foods and nutraceuticals in the primary prevention of cardiovascular diseases. J Nutr Metab 2012: 569486, 2012.

20. Olsen RL, Toppe J and Karunasagar I: Challenges and realistic opportunities in the use of by-products from processing of fish and shellfish. Trends Food Sci Technol 36: 144-151, 2014.

21. Palomares O, Akdis M, Martín-Fontecha M and Akdis CA: Mechanisms of immune regulation in allergic diseases: The role of regulatory T and B cells. Immunol Rev 278: 219-236, 2017.

22. Kubo M: Innate and adaptive type 2 immunity in lung allergic inflammation. Immunol Rev 278: 162-172, 2017.

23. Asano K, Yoshimura S and Nakane A: Alteration of intestinal microbiota in mice orally administered with salmon cartilage proteoglycan, a prophylactic agent. PLoS One 8, e75008, 2013. 